\title{
Ganhos realizados com a seleção para caracteres de importância agronômica em cenoura
}

\author{
Giovani Olegário da Silva; Jairo V Vieira \\ Embrapa Hortaliças, C. Postal 218, 70359-970 Brasília-DF; olegario@cnph.embrapa.br
}

\begin{abstract}
RESUMO
A estimativa do progresso genético é de importância em programas de melhoramento para orientar o melhorista sobre a eficiência dos métodos de seleção empregados para as características de interesse nas populações sob seleção. O objetivo do trabalho foi verificar os ganhos reais com a seleção para caracteres de importância agronômica em uma população de cenoura em sucessivas gerações de seleção. O experimento foi conduzido na Embrapa Hortaliças, Brasília. Uma população de cenoura foi selecionada por seis gerações consecutivas nos verões de 1999 a 2004. No verão de 2005, amostras de sementes provenientes de cada ano foram semeadas a campo em delineamento de blocos casualizados com cinco repetições e parcelas de $1 \mathrm{~m}^{2}$. Aos 60 e 90 dias após a semeadura foram realizadas avaliações para severidade de "queima-das-folhas", e aos 90 dias as raízes foram colhidas e avaliadas, em cada parcela, para as características número e massa de raízes refugo, número e massa de raízes comerciais com ombro verde, número e massa de raízes comerciais sem ombro verde, número, massa e massa média total de raízes, e número, massa e massa média de raízes comerciais. Foram realizadas análise de variância e comparações de médias entre os tratamentos por Scott-Knott, e obtidos os ganhos reais com a seleção. Houve ganhos superiores apenas nos primeiros ciclos de seleção para diminuição das raízes refugo e o aumento da resistência à queima-das-folhas. A seleção para queima-das-folhas só foi eficiente aos 90 dias. A quantidade de raízes classificadas como comerciais aumentou durante todos os ciclos de seleção, tanto com ombro quanto sem ombro verde, sendo que o ganho foi maior para as raízes sem ombro verde.
\end{abstract}

Palavras-chave: Daucus carota L., seleção recorrente, progresso genético.

\begin{abstract}
Gain obtained by the selection for agronomic traits in carrot

The genetic progress estimative is an important aspect in breeding programs, in order to evaluate the breeding selection methods efficiency for the characteristics of interest in the populations under selection. In this research, the gains obtained from the selection of agronomic traits were evaluated in a carrot population through successive generations. The experiment was performed at Embrapa Hortaliças, Brasília, Brazil. A carrot population was selected every summer during six consecutive generations from 1999 to 2004. In the summer of 2005, samples of seeds harvested in the previous years were sown in plots of $1 \mathrm{~m} 2$ in randomized blocks design with five replications. The plants were evaluated for severity of leaf blight at 60 and 90 days after sowing and harvested at 90 days after sowing when each plot was evaluated for the characters of number and mass of waste roots, number and mass of marketable roots with green shoulder, number and mass of marketable roots without green shoulder, number, mass and average mass of total roots and number, mass and average mass of marketable roots. The data were submitted to analyses of variance, and comparisons of treatment means by ScottKnott, and the gain obtained with selection was calculated. Superior gains occurred only in the first cycles of selection towards the decrease of refused roots and increase of resistance to leaf blight. The selection for leaf blight resistance was efficient only when performed at 90 days after sowing, because the disease severity was low at 60 days. The amount of roots classified as marketable with and without green shoulder increased during all the selection cycles, although the gains were higher for the roots without green shoulder.
\end{abstract}

Keywords: Daucus carota L., recurrent selection, genetic progress.

(Recebido para publicação em 21 de maio de 2008; aceito em 3 de agosto de 2009) (Received in May 21, 2008; accepted in August 3, 2009)

\begin{abstract}
A cenoura é uma importante fonte de $\beta$-catoreno para a alimentação humana. O melhoramento genético tem muitos objetivos como a busca por resistência a doenças, maior produtividade e uniformidade das raízes, melhorias nos caracteres de aparência das raízes, quantidade de nutrientes, dentre outros. A seleção aplicada a todas estas características busca o avanço em direção aos objetivos que o melhorista deseja. Porém, a confirmação da eficiência desta seleção deve ser monitorada para
\end{abstract}

se saber se os objetivos estão sendo alcançados (Barbosa Neto et al., 2000; Cruz et al., 2004).

O progresso genético obtido pela seleção está associado à existência de variabilidade genética, à seleção natural e/ou artificial e ao ajuste dos genótipos aos ambientes existentes. Comprovada a presença da variabilidade genética e, sobretudo o valor desta em relação à variação não-genética, a seleção assume grande importância no progresso genético. A seleção objetiva acumular alelos favoráveis à característica de interesse em determinada população e é um processo vinculado a uma constante e permanente renovação (Reis et al., 2004).

De acordo com a estratégia de seleção e o ganho que ela proporcionará para cada característica, pode-se orientar, de maneira mais efetiva, a melhor estratégia de melhoramento que permitam os ganhos desejados para as características de interesse no programa de melhoramento (Cruz et al., 2004). 
Os progressos genéticos referem-se às alterações observadas nas características de interesse, durante um ciclo de seleção, com a recombinação e multiplicação das unidades selecionadas. Tais modificações ocorrerão em magnitude e sentido variados, dependendo da estratégia e dos critérios de seleção adotados. Assim, uma das atribuições mais importantes do melhorista é identificar critérios de seleção capazes de promover alterações, no sentido desejado, nas características de interesse dentro de um programa de melhoramento (Reis et al., 2004).

O progresso genético é um aspecto de fundamental importância em programas de melhoramento. Sua estimativa fornece uma oportunidade de correlacionar ganhos alcançados com os métodos de melhoramento empregados, possibilitando o ajuste dos objetivos propostos inicialmente (Barbosa Neto et al., 2000). Desta forma, o constante monitoramento do progresso obtido pela seleção nos programas de melhoramento é necessário, de forma a orientar o melhorista sobre a eficiência dos métodos de seleção empregados para as características de interesse nas populações sob seleção, justificando a realização de trabalhos como este proposto.

A verificação do progresso genético obtido com o melhoramento é tema de vários trabalhos na literatura, com diversas culturas, porém, na literatura são comparadas cultivares ou linhagens antigas com cultivares ou linhagens modernas (Breseghello et al., 1999; Barbosa Neto et al., 2004; Tabiena et al., 2008). Não há registro de trabalhos de acompanhamento do progresso genético de determinada população de cenoura durante sucessivos ciclos de seleção. No entanto, Miranda et al. (1977), analisando uma população de milho selecionada por oito ciclos de seleção, verificaram incremento de $1,90 \%$ por ciclo para rendimento de grãos, porém ganhos não foram obtidos para porcentagem de plantas acamadas e quebradas. Em milho pipoca, Freitas Júnior et al. (2009) constataram a ocorrência de sucessivos ganhos na população UNB após quatro ciclos de seleção recorrente, para as principais características comerciais da cultura, sem perda da variabilidade genética com a concentração de alelos favoráveis (Vilela et al., 2008). Todavia, os ganhos para rendimento de grãos não foram uniformes entre os ciclos e houve aumento praticamente desprezível do segundo para o terceiro ciclo.

O objetivo deste trabalho foi verificar os ganhos reais com a seleção para caracteres de importância agronômica em uma população de cenoura em sucessivas gerações de seleção.

\section{MATERIAL E MÉTODOS}

O ensaio foi conduzido no campo experimental da Embrapa Hortaliças no Distrito Federal, em Latossolo Vermelho Amarelo com textura média (32\% de argila) (Embrapa, 1999), preparado com encanteirador após subsolagem e gradagem. Uma população de cenoura foi avaliada e selecionada por seis gerações consecutivas nos verões de 1999, 2000, 2001, 2002, 2003 e 2004, obtida por seleção recorrente entre e dentro de famílias de meios-irmãos, pela utilização de níveis independentes de eliminação e índice de seleção baseado no ganho desejado.

Parte das sementes de cada ano foi armazenada em câmara seca a $6^{\circ} \mathrm{C}$ e umidade de $50 \%$, em embalagens Polche de alumínio. Foi realizada a determinação da germinação das sementes provenientes de cada geração em laboratório. No verão de 2005, seis amostras de sementes provenientes de cada ano, foram semeadas a campo em delineamento de blocos casualizados com cinco repetições em parcelas de $1 \mathrm{~m}^{2}$. A semeadura foi feita em quatro linhas transversais ao comprimento do canteiro, espaçadas de $25 \mathrm{~cm}$ uma da outra, totalizando aproximadamente cerca de 100 plantas por $\mathrm{m}^{2}$. A quantidade de sementes do ano de 1999 foi aumentada corrigindo-se o fator porcentagem de germinação para 95\%. O desbaste foi realizado 30 dias após semeio, de modo que os espaçamentos entre plantas foi de $2 \mathrm{~cm}$ e entre linhas de $20 \mathrm{~cm}$.

Aos 60 e 90 dias após a semeadura foram realizadas avaliações para severidade de "queima-das-folhas" nas parcelas, utilizando-se uma adaptação da escala de notas de Aguilar et al. (1986), variando de 1 a 5, em que: 1= parcelas com sintomas severos, ou seja, todas as folhas da parcela totalmente necrosadas pela presença do patógeno e $5=$ parcelas isentas de sintomas. As notas de severidade em cada parcela foram atribuídas em conjunto por dois avaliadores. Aos 90 dias após semeio as raízes foram colhidas e avaliadas em cada parcela para as características número de raízes refugo, massa de raízes refugo, número de raízes comerciais com ombro verde, massa de raízes comerciais com ombro verde, número de raízes comerciais sem ombro verde, massa de raízes comerciais sem ombro verde, número total de raízes, massa total de raízes, massa média total de raízes, número de raízes comerciais, massa de raízes comerciais e massa média de raízes comerciais. Foram consideradas raízes comerciais para mesa, aquelas com comprimento entre 20 e $25 \mathrm{~cm}$ e sem defeitos fisiológicos como rachadura e embonecamento.

Os dados foram submetidos à análise de homogeneidade de variância (teste de Bartllet) e de normalidade (Lilliefors). Os caracteres número e massa de raízes com ombro verde foram transformados por Log (x), para atender a pressuposição de normalidade de distribuição. Posteriormente foi realizada análise de variância, comparações de médias entre os tratamentos por Scott-Knott (1974), e obtidos os ganhos reais com a seleção, pela diferença de desempenho dos diferentes tratamentos em relação à população inicial do ano de 1999. Todas as operações estatísticas foram realizadas utilizando-se o aplicativo computacional Genes (Cruz, 2006).

\section{RESULTADOS E DISCUSSÃO}

Pela análise de variância, com exceção de número total de raízes por parcela e "queima-das-folhas" aos 60 dias, os demais caracteres expressaram significância, indicando que o desempenho dos diferentes tratamentos entre as gerações de seleção foram divergentes.

O caracter número total de raízes por parcela não revelou diferença significativa, o que já era esperado, uma vez que os espaçamentos entre plantas e entre linhas foram os mesmos para todos os tratamentos, e o fator porcentagem de 
Tabela 1. Comparação entre as médias entre seis tratamentos avaliados durante seleção recorrente de 1999 a 2004 para uma população de cenoura em fase de melhoramento por Scott-Knott (1974) (average comparison among six treatments evaluated through recurrent selection from 1999 to 2004 for a carrot population in breeding phase by Scott-Knott (1974)). Brasília, Embrapa Hortaliças, 2007.

\begin{tabular}{lccccccc}
\hline & NRR $^{1}$ & MRR & NRCCOV & MRCCOV & NRCSOV & MRCSOV & NTR \\
\hline 1999 & $87,60 \mathrm{a}^{*}$ & $1750 \mathrm{a}$ & $19.20 \mathrm{~b}$ & $999 \mathrm{c}$ & $22,00 \mathrm{~b}$ & $1228 \mathrm{c}$ & $128,80 \mathrm{a}$ \\
2000 & $84,60 \mathrm{a}$ & $1746 \mathrm{a}$ & $22.00 \mathrm{~b}$ & $1298 \mathrm{c}$ & $28,80 \mathrm{~b}$ & $1380 \mathrm{c}$ & $135,40 \mathrm{a}$ \\
2001 & $71,20 \mathrm{~b}$ & $1346 \mathrm{~b}$ & $28.60 \mathrm{~b}$ & $1726 \mathrm{~b}$ & $30,40 \mathrm{~b}$ & $1602 \mathrm{c}$ & $130,20 \mathrm{a}$ \\
2002 & $74,00 \mathrm{~b}$ & $1330 \mathrm{~b}$ & $27.40 \mathrm{~b}$ & $1496 \mathrm{~b}$ & $37,80 \mathrm{~b}$ & $1884 \mathrm{~b}$ & $139,20 \mathrm{a}$ \\
2003 & $67,20 \mathrm{~b}$ & $1116 \mathrm{~b}$ & $24.40 \mathrm{~b}$ & $1434 \mathrm{a}$ & $35,60 \mathrm{~b}$ & $1998 \mathrm{~b}$ & $127,20 \mathrm{a}$ \\
2004 & $54,20 \mathrm{~b}$ & $1036 \mathrm{~b}$ & $31.80 \mathrm{a}$ & $2018 \mathrm{a}$ & $51,20 \mathrm{a}$ & $3140 \mathrm{a}$ & $137,20 \mathrm{a}$ \\
\hline & MTR & MMTR & NRC & MRC & MMRC & QF60 & QF90 \\
\hline 1999 & $3977 \mathrm{~b}$ & $31,27 \mathrm{~b}$ & $41,20 \mathrm{c}$ & $2227 \mathrm{c}$ & $54,19 \mathrm{~b}$ & $3,50 \mathrm{a}$ & $2,70 \mathrm{~b}$ \\
2000 & $4424 \mathrm{~b}$ & $33,10 \mathrm{~b}$ & $50,80 \mathrm{c}$ & $2678 \mathrm{c}$ & $52,78 \mathrm{~b}$ & $3,70 \mathrm{a}$ & $2,90 \mathrm{a}$ \\
2001 & $4674 \mathrm{~b}$ & $36,44 \mathrm{~b}$ & $59,00 \mathrm{~b}$ & $3328 \mathrm{~b}$ & $56,52 \mathrm{~b}$ & $3,80 \mathrm{a}$ & $3,00 \mathrm{a}$ \\
2002 & $4710 \mathrm{~b}$ & $34,39 \mathrm{~b}$ & $65,20 \mathrm{~b}$ & $3380 \mathrm{~b}$ & $52,01 \mathrm{~b}$ & $3,50 \mathrm{a}$ & $3,00 \mathrm{a}$ \\
2003 & $4548 \mathrm{~b}$ & $36,93 \mathrm{~b}$ & $60,00 \mathrm{~b}$ & $3432 \mathrm{~b}$ & $57,65 \mathrm{a}$ & $3,50 \mathrm{a}$ & $3,00 \mathrm{a}$ \\
2004 & $6194 \mathrm{a}$ & $46,17 \mathrm{a}$ & $83,00 \mathrm{a}$ & $5158 \mathrm{a}$ & $62,65 \mathrm{a}$ & $3,60 \mathrm{a}$ & $3,00 \mathrm{a}$ \\
\hline
\end{tabular}

${ }^{1} \mathrm{NRR}=$ número de raízes refugo; $\mathrm{MRR}=$ massa de raízes refugo $(\mathrm{g})$; $\mathrm{NRCCOV}=$ número de raízes comerciais com ombro verde; $\mathrm{MRC}$ $\mathrm{COV}=$ massa de raízes comerciais com ombro verde $(\mathrm{g})$; $\mathrm{NRCSOV}=$ número de raízes comerciais sem ombro verde; $\mathrm{MRCSOV}=$ massa de raízes comerciais sem ombro verde $(\mathrm{g}) ; \mathrm{NTR}=$ número total de raízes; $\mathrm{MTR}=$ massa total de raízes $(\mathrm{g})$; $\mathrm{MMTR}=$ massa média total de raízes $(\mathrm{g})$; $\mathrm{NRC}=$ número de raízes comerciais; $\mathrm{MRC}=$ massa de raízes comercias (g); $\mathrm{MMRC}=$ massa média de raízes comerciais $(\mathrm{g})$; QF60= queima-das-folhas aos 60 dias após semeadura (notas de 1 a 5) e QF90= queima-das-folhas aos 90 dias após semeadura (notas de 1 a 5$)\left({ }^{1} \mathrm{NRR}=\right.$ number of refused roots; $\mathrm{MRR}=$ mass of refused roots $(\mathrm{g})$; NRCCOV= number of commercial roots with green shoulder; MRCCOV= mass of commercial roots with green shoulder $(\mathrm{g})$; NRCSOV= number of commercial roots without green shoulder; $\mathrm{MRCSOV}=$ mass of commercial roots without green shoulder $(\mathrm{g})$; NTR $=$ number of total roots; MTR = mass of total roots $(\mathrm{g})$; MMTR= average mass of total roots $(\mathrm{g})$; $\mathrm{NRC}=$ number of commercial roots; $\mathrm{MRC}=$ mass of commercial roots $(\mathrm{g})$; MMRC $=$ average mass of commercial roots (g); QF60= leaf blight, 60 days after sowing (notes from 1 to 5) and QF90= leaf blight, 90 days after sowing (notes from 1 to 5)). *Médias seguidas de letras diferentes na coluna diferenciam-se significativamente a $5 \%$ de probabilidade (*means followed by different letters in the column differ significantly $5 \%$ of probability).

germinação foi corrigido pela quantidade de sementes do ano de 1999. No entanto, esse resultado foi importante, pois confirmou que o efeito do tempo diferenciado de armazenamento não afetou o estande de plantas para os demais anos.

Para o caracter "queima-das-folhas" aos 60 dias após semeadura, a ausência de significância estatística das diferenças entre os tratamentos, indica que a ocorrência natural naquele momento não era intensa o suficiente para discriminar os diferentes níveis de resistência. Isto pode ser verificado comparando-se com a avaliação aos 90 dias, que revelou significância e, portanto, possibilitou a diferenciação entre os tratamentos. A maior severidade da doença aos 90 dias após semeadura também pode ser verificada pelos valores das médias, em que para a avaliação aos 60 e 90 dias foram de 3,60 e 2,93, respectivamente.

Pelas comparações das médias (Tabela 1), verifica-se que com o passar dos ciclos de seleção, ganhos puderam ser obtidos em relação à diminuição das raízes consideradas refugo, tanto em massa quanto em número. No entanto, significativamente, as diferenças ocorreram apenas nos primeiros ciclos de seleção. $\mathrm{O}$ mesmo ocorreu para "queima-dasfolhas" aos 90 dias. Isto indica que para estes caracteres a variabilidade genética em relação à fenotípica era maior nos primeiros ciclos de seleção.

O número total de raízes não se alterou com os ciclos de seleção, pois como foi discutido, os espaçamentos entre e dentro de linhas foram os mesmos para todos os tratamentos. No entanto, a massa total e a massa média de raízes aumentaram no intervalo entre 1999 até 2004, o que equivale a preconizar que as parcelas passaram a render mais (Tabela 1).

Tanto para número, massa total e massa média de raízes com características comerciais quanto para massa de raízes comerciais sem ombro verde aumentou no decorrer dos ciclos de seleção. Por outro lado, o número e a massa de raízes comerciais com ombro verde também aumentaram, indicando que a seleção para diminuição de ombro verde não foi tão efetiva quanto à realizada para aumento das raízes comerciais (Tabela 1). Ombro verde é conhecidamente um caracter de forte influência ambiental e de difícil seleção (Traka-Mavrona, 1996). No entanto, pelos ganhos em porcentagem em relação à população inicial do ano de 1999 (Tabela 2), é possível verificar que maiores ganhos foram obtidos para maior massa e número de raízes comerciais, tanto no total quanto com e sem ombro verde, quase todos acima de $100 \%$. De todo modo, o aumento das raízes comerciais sem ombro verde foi maior que com ombro verde, indicando que houve progresso com a seleção para este caracter.

Apesar de não haver relatos na literatura de trabalhos semelhantes com cenoura para os caracteres avaliados, 
Tabela 2. Porcentagem de aumento ou decréscimo das médias dos caracteres em sucessivos ciclos de seleção, relativamente ao desempenho inicial em 1999 de uma população de cenoura em fase de melhoramento. (Percentage of increase or decrease of the characters means in successive selection cycles, relatively to the initial perform in 1999 of a carrot population in breeding phase). Brasília, Embrapa Hortaliças, 2007.

\begin{tabular}{lrrrrrrr}
\hline & NRR & MRR & NRCCOV & MRCCOV & NRCSOV & MRCSOV & NTR \\
\hline 1999 & 100,00 & 100,00 & 100,00 & 100,00 & 100,00 & 100,00 & 100,00 \\
$1999-2000$ & 96,58 & 99,77 & 114,58 & 129,93 & 130,91 & 112,38 & 105,12 \\
$1999-2001$ & 81,28 & 76,91 & 148,96 & 172,77 & 138,18 & 130,46 & 101,09 \\
$1999-2002$ & 84,47 & 76,00 & 142,71 & 149,75 & 171,82 & 153,42 & 108,07 \\
$1999-2003$ & 76,71 & 63,77 & 127,08 & 143,54 & 161,82 & 162,7 & 98,76 \\
$1999-2004$ & 61,87 & 59,2 & 165,63 & 202,00 & 232,73 & 255,7 & 106,52 \\
\hline & MTR & MMTR & NRC & MRC & MMRC & QF60 & QF90 \\
\hline 1999 & 100,00 & 100,00 & 100,00 & 100,00 & 100,00 & 100,00 & 100,00 \\
$1999-2000$ & 111,24 & 105,85 & 123,3 & 120,25 & 97,41 & 105,71 & 107,41 \\
$1999-2001$ & 117,53 & 116,54 & 143,2 & 149,44 & 107,09 & 108,57 & 111,11 \\
$1999-2002$ & 118,43 & 109,97 & 158,25 & 151,77 & 92,01 & 100,00 & 111,11 \\
$1999-2003$ & 114,36 & 118,11 & 145,63 & 154,11 & 110,86 & 100,00 & 111,11 \\
$1999-2004$ & 155,75 & 147,64 & 201,46 & 231,61 & 108,67 & 102,86 & 111,11 \\
\hline
\end{tabular}

$\mathrm{NRR}=$ número de raízes refugo; $\mathrm{MRR}=$ massa de raízes refugo $(\mathrm{g})$; $\mathrm{NRCCOV}=$ número de raízes comerciais com ombro verde; $\mathrm{MRCCOV}=$ massa de raízes comerciais com ombro verde $(\mathrm{g})$; $\mathrm{NRCSOV}=$ número de raízes comerciais sem ombro verde; $\mathrm{MRCSOV}=$ massa de raízes comerciais sem ombro verde $(\mathrm{g})$; NTR= número total de raízes; MTR= massa total de raízes $(\mathrm{g})$; MMTR= massa média total de raízes (g); $\mathrm{NRC}=$ número de raízes comerciais; $\mathrm{MRC}$; massa de raízes comerciais $(\mathrm{g})$; MMRC = massa média de raízes comerciais $(\mathrm{g})$; $\mathrm{QF60=}$ queimadas-folhas aos 60 dias após semeadura (notas de 1 a 5) e QF90= queima-das-folhas aos 90 dias após semeadura (notas de 1 a 5) [(NRR= number of refused roots; $\mathrm{MRR}=$ mass of refused roots $(\mathrm{g})$; $\mathrm{NRCCOV}=$ number of commercial roots with green shoulder; $\mathrm{MRCCOV}=$ mass of commercial roots with green shoulder $(\mathrm{g})$; NRCSOV= number of commercial roots without green shoulder; MRCSOV= mass of commercial roots without green shoulder $(\mathrm{g})$; NTR= number of total roots; MTR= mass of total roots $(\mathrm{g})$; MMTR= average mass of total roots $(\mathrm{g})=\mathrm{NRC}$ : number of commercial roots; $\mathrm{MRC}=$ mass of commercial roots $(\mathrm{g}) ; \mathrm{MMRC}=$ average mass of commercial roots $(\mathrm{g}) ; \mathrm{QF} 60=\mathrm{leaf}$ blight, 60 days after sowing (notes from 1 to 5) and QF90= leaf blight, 90 days after sowing (notes from 1 to 5)].

este tipo de experimento é muito importante na quantificação dos ganhos genéticos obtidos pelos métodos de melhoramento empregados para cada caracter. Do mesmo modo, Miranda et al. (1977) analisando uma população de milho selecionada por oito ciclos de seleção, verificaram incremento de $1,90 \%$ por ciclo para rendimento de grãos, porém ganhos não foram obtidos para porcentagem de plantas acamadas e quebradas. De forma análoga, Freitas Júnior et al. (2009) constataram ganhos para rendimento de grãos em seleção recorrente com a população de milho pipoca UNB; porém o avanço foi ínfimo do segundo para o terceiro ciclo de seleção recorrente intrapopulacional.

Não se pode desconsiderar o fator tempo de armazenamento das sementes, que apesar de ser realizado em condições adequadas, pode proporcionar pequena diferença de vigor aos diferentes tratamentos. A existência ou não de influência significativa deste fator nos diferentes caracteres a campo pode ser alvo de futuras pesquisas. Porém, quanto ao estande final, pôde-se, neste trabalho, verificar que não houve influência do vigor da semente.

Desta forma, houve ganhos superiores apenas nos primeiros ciclos de seleção para os caracteres diminuição das raízes refugo e o aumento da resistência à "queima-das-folhas", o que indica que para estes caracteres a variabilidade genética em relação à fenotípica era maior nos primeiros ciclos de seleção; a seleção para "queimadas-folhas" só foi eficiente aos 90 dias, pois aos 60 dias o nível de severidade da doença foi baixo, sendo que o final do ciclo pode ter influenciado para o aumento da severidade dos sintomas; o número, a massa e a massa média de raízes com características comerciais aumentaram no decorrer dos ciclos de seleção, caracterizando ganho positivo. Verificou-se ainda que a quantidade de raízes classificadas como comerciais aumentou durante todos os ciclos de seleção, tanto com ombro verde quanto sem ombro verde, porém o ganho foi maior para as raízes sem ombro verde, indicando que a seleção foi efetiva para este caracter.

\section{REFERÊNCIAS}

AGUILAR JAE; REIFSCHNEIDER FJB; ROSSI PFE; DELLA VECCHIA PT. 1986. Nível de resistência de cenoura a Alternaria dauci e interação com tratamento químico. Horticultura Brasileira 4: 19-22.

BARBOSA NETO JF; MATIELLO RR; CARVALHO FIF; OLIVEIRA JMS; PEGORARO DG; SCHNEIDER F; SORDI MEB; VACARO E. 2000. Progresso genético no melhoramento da aveia-branca no Sul do Brasil. Pesquisa Agropecuária Brasileira 35: 1605-1612.

BRESEGHELLO F; RANGEL, PHN; MORAIS ON. 1999. Ganho de produtividade pelo melhoramento genético do arroz irrigado no Nordeste do Brasil. Pesquisa Agropecuária Brasileira 34: 399-407.

CRUZ CD. 2006. Programa Genes: aplicativo computacional em genética e estatística. Viçosa: UFV. 442p.

CRUZ CD; REGAZZI AJ; CARNEIRO PCS. 2004. Modelos biométricos aplicados ao 
melhoramento genético. Viçosa: UFV. 480p.

EMBRAPA SOLOS. 1999. Sistema brasileiro de classificação de solos. Brasília: EMBRAPA. Produção de Informação. 412p.

FREITAS JÚNIOR SP; AMARAL JÚNIOR AT; RANGEL, RM; VIANA AP. 2009. Genetic gains in popcorn by full-sib recurrent selection. Crop Breeding and Applied Biotechnology 9: 1-7.

MIRANDA LT; MIRANDA LEC; POMMER CV; SAWAZAKI E. 1977. Oito ciclos de seleção entre e dentro de famílias de meios irmãos no milho IAC-1. Bragantia 36: 177-196.

REIS EF; REIS MS; CRUZ CD; SEDIYAMA T. 2004. Comparação de procedimentos de seleção para produção de grãos em populações de soja. Ciência Rural 34: 685-692.

SCOTT AJ; KNOTT MA. 1974. A cluster analysis method for grouping means in the analysis of variance. Biometrics 30: 507-512.

TABIENA RE; SAMONTEA SOPB; MCCLUNGB AM. 2008. Forty-eight years of rice improvement in Texas since the release of cultivar Bluebonnet in 1944. Crop Science
48: 2097-2106

TRAKA-MAVRONA E. 1996. Effects of competition on phenotypic expression and differentiation of five quality traits of carrot (Daucus carota L.) and their implications in breeding. Scientia Horticulturae 65: $335-$ 340.

VILELA FO; AMARAL JÚNIOR AT; PEREIRA MG; SCAPIM CA; VIANA AP; FREITAS JÚNIOR SP. 2008. Effect of recurrent selection on the genetic variability of the UNB-2U popcorn population. Acta Scientiarum. Agronomy 30: 25-30. 